\title{
Correlation between osteoarthritis and monocyte chemotactic protein-1 expression: a meta-analysis
}

\author{
Feifei $\mathrm{Ni}^{1} \mathbb{B}$, Yanchao Zhang ${ }^{2}$, Xiaoxiao Peng ${ }^{3}$ and Jianjun $\mathrm{Li}^{i^{*}}$
}

\begin{abstract}
Objective: We evaluated the association between monocyte chemotactic protein-1 (MCP-1) and osteoarthritis.

Methods: We searched PubMed, Cochrane Library, Embase, Web of Science, China National Knowledge Infrastructure (CNKI), VIP (Chinese database), and Wan Fang (Chinese database) (before May 10, 2020), with no language limitations. STATA version 12.0 and Revman version 5.3 were used for data analysis. The standard mean difference (SMD) and corresponding 95\% confidence intervals (95\% Cls) were calculated. Nine clinical studies, including 376 patients with osteoarthritis and 306 healthy controls, were evaluated.
\end{abstract}

Results: The combined SMDs of MCP-1 expression levels suggested that MCP-1 expression was significantly higher in patients with osteoarthritis than healthy controls (SMD $=1.97,95 \% \mathrm{Cl}=0.66-3.28, p=0.003)$. Moreover, subgroup analysis implied that osteoarthritis patients from both Asians and mixed populations had higher MCP-1 expression levels than controls, whereas Caucasians did not ( $p>0.05)$. Serum MCP-1 levels (SMD $=2.83,95 \% \mathrm{Cl}=$ 1.07-4.6, $p<0.00001$ ) were significantly higher in patients with osteoarthritis than in controls; however, this difference was not significant in synovial fluid and cartilage tissue. Subgroup analysis for ethnicity showed that MCP-1 levels were significantly higher in Chinese, Dutch, and Brazilian patients with osteoarthritis than in control groups, although significant differences were not observed for American and Italian subgroups.

Conclusions: Our meta-analysis demonstrated that MCP-1 expression levels were higher in patients with osteoarthritis than in healthy controls and that MCP-1 may play important roles in the progression of osteoarthritis. Serum MCP-1 levels may serve as a potential biomarker for the diagnosis of osteoarthritis.

Keywords: Osteoarthritis, Monocyte chemotactic protein-1, Meta-analysis

\section{Introduction}

Osteoarthritis (OA) is a type of degenerative articular cartilage disease related to inflammation and is characterized by joint stiffness and pain. OA is most prevalent in middle-aged and elderly individuals, and its incidence has increased in recent years. In patients with OA, joint activity is significantly reduced. If not effectively

\footnotetext{
* Correspondence: Chinan888999@163.com

${ }^{1}$ Department of Orthopaedics, Shengjing Hospital of China Medical

University, Sanhao Street No. 36, Heping District, Shenyang 110004, Liaoning, People's Republic of China

Full list of author information is available at the end of the article
}

controlled in a timely manner, OA can cause limb dysfunction and affect the patient's quality of life [1]. The incidence of osteoarthritis varies in different joints; the most common joint to be affected is the knee, followed by the hip; epidemiological studies have found that the global incidence of $\mathrm{OA}$ in the knee is $3.8 \%$, whereas that in the hip is $0.85 \%$ [2]. In contrast, the incidence of knee OA in China is $8.1 \%$, whereas that in the United States of America (USA) is $12.1 \%$, which is similar to that in Europe; such incidence is much lower (5.5\%) in India [3]. There are currently no curative treatments for OA,

C C The Author(s). 2020 Open Access This article is licensed under a Creative Commons Attribution 4.0 International License, which permits use, sharing, adaptation, distribution and reproduction in any medium or format, as long as you give appropriate credit to the original author(s) and the source, provide a link to the Creative Commons licence, and indicate if changes were made. The images or other third party material in this article are included in the article's Creative Commons licence, unless indicated otherwise in a credit line to the material. If material is not included in the article's Creative Commons licence and your intended use is not permitted by statutory regulation or exceeds the permitted use, you will need to obtain permission directly from the copyright holder. To view a copy of this licence, visit http://creativecommons.org/licenses/by/4.0/. The Creative Commons Public Domain Dedication waiver (http://creativecommons.org/publicdomain/zero/1.0/) applies to the data made available in this article, unless otherwise stated in a credit line to the data. 
resulting in heavy economic burden on patients, families, and society. Additionally, the molecular mechanisms of $\mathrm{OA}$ are unclear, and new and effective biomarkers are urgently needed to improve methods for the diagnosis and prevention of OA occurrence and progression [4]. Inflammatory mechanisms are important factors promoting the development of OA. In OA, cytokine and chemokine production, the synovium response, cell infiltration, and inflammatory pathway activation affect disease progression and have been observed in animal models. Chemokines are inducible secreted proinflammatory cytokines with a relative molecular mass of approximately $8-10 \mathrm{kDa}$. The main function of chemokines is to stimulate different types of cells, including neutrophils, monocytes, lymphocytes, and fibroblasts, to undergo chemotaxis, thereby mediating cell aggregation and activation at the site of inflammation and facilitating tissue damage and repair. These molecules also play important roles in various biological processes, such as immune surveillance, organ development, angiogenesis, and immune responses [5-7]. Importantly, many experimental studies have demonstrated that chemokines are involved in the pathogenesis of OA and may be new targets for the early intervention and treatment of OA [8-12].

MCP-1 is an important chemokine secreted by synovial fibroblasts in response to stimulation by the inflammatory cytokines interleukin (IL)-1, tumor necrosis factor- $\alpha$, and interferon- $\gamma$ [13]. Notably, MCP- 1 is expressed in chondrocytes, osteoblasts, synovial cells, and other cells and plays pivotal roles in bone metabolism and OA [14]. MCP-1 has also been shown to be highly expressed in many diseases [15-19], including $\mathrm{OA}$ and rheumatoid arthritis [20, 21]. Additionally, MCP-1 attracts monocytes, resulting in accumulation of monocytes and secretion of cell products to facilitate the OA immune response; this leads to clinical symptoms, such as redness, swelling, and pain [22]. Moreover, abnormal expression of $\mathrm{MCP}-1$ can promote the transformation of monocytes into macrophages in the knee joint capsule, stimulate osteoclasts for bone absorption, induce inflammation, and accelerate the development of $\mathrm{OA}$, leading to joint destruction [23, 24]. MCP-1 also activates monocytes and macrophages to release IL-1 and IL- 6 and promotes the production of chemokines and pro-inflammatory cytokines through autocrine and paracrine feedback loops [25]. Reducing the levels of MCP-1 in the joint fluid of patients with OA can alleviate damage to articular cartilage, inhibit the transformation and activation of macrophages, and maintain the stability of the local microenvironment of the joint. Thus, these findings suggest that MCP-1 is closely related to OA; however, some other studies have shown that there is no obvious relationship between MCP-1 expression and OA
[26-29]. Thus, the role of MCP-1 in OA remains controversial.

Therefore, in this study, we conducted a meta-analysis to assess the relationship between MCP-1 expression and $\mathrm{OA}$.

\section{Materials and methods \\ Literature search}

We searched the following electronic databases without any language restrictions: PubMed, Cochrane Library, Embase, Web of Science, Chinese National Knowledge Infrastructure, VIP, and Wan Fang. The search strategy showed high sensitivity using combinations of the following keywords and MeSH terms: "CCL2" or "MCP-1" or "monocyte chemotactic and activating factor" or "monocyte chemoattractant protein-1" and "Osteoarthritis" or "Osteoarthritis, Spine" or "Osteoarthritis, Knee" or "Osteoarthritis, Hip" or "Knee osteoarthritis" or "Spine osteoarthritis" or "Spinal osteoarthritis" or "Lumbar osteoarthritis" or "Coxarthrosis."

\section{Selection criteria}

The study selection criteria were as follows: (1) only case-control or cross-sectional studies in the population to explore the relationship between MCP-1 and OA were included, (2) patients in the studies must have met the diagnostic criteria for OA, (3) studies provided means and standard deviations or means and standard errors of MCP-1 levels in patients with OA and healthy controls, and (4) studies must have had sufficient and original data. Studies that did not meet the selection criteria were excluded. If one author published different studies on the same topic, the most recently published study or the study with the largest sample size was selected.

\section{Data extraction}

From the selected articles, two researchers (Feifei Ni, Xiaoxiao Peng) independently extracted and recorded the required information. Disagreements over data or included studies were agreed upon through discussion of all items. The recorded information included surnames of initial authors, region, language, publication year, patient age, MCP-1 detection method, source of sample, and MCP-1 levels in cases and controls.

\section{Quality of the studies}

Two observers (Feifei Ni, Xiaoxiao Peng) used the Newcastle-Ottawa Scale (NOS) to assess the quality of the included studies [30]. The NOS consisted of three factors: (1) patient selection, 0-4; (2) comparability of patients, 0-2; and (3) clinical outcomes, 0-3. NOS scores ranged from 0 to 9 , and the quality of the included studies was then categorized as low quality (0-6) 
or high quality (7-9). When there were disagreements or discrepancies with regard to NOS scores between the two researchers, we sought assistance from a third researcher.

\section{Statistical analysis}

The relationship between MCP-1 levels and OA susceptibility was assessed using standardized mean differences (SMDs) and 95\% confidence intervals (95\% CIs). We used Cochran's $Q$ test (results displaying $p<0.05$ were considered significant) and $I^{2}$ tests to quantify heterogeneity among studies [31]. A random-effects model was used when there was significant heterogeneity $(p<0.05$ or $I^{2}>50 \%$ ), whereas SMDs were pooled based on a fixed-effects model [32]. When there was significant heterogeneity, subgroup analysis was performed to identify the potential reasons for the differences in MCP-1 levels between patients with $\mathrm{OA}$ and controls. In addition, sensitivity analysis was used to assess whether a single study had an impact on the entire assessment, and the impact of publication bias was analyzed using Egger's test (results displaying $p<0.05$ were considered significant), which can be used to evaluate asymmetry visible in a funnel plot [33, 34]. The data were analyzed with the software programs Review Manager 5.3 and STATA version 12.0. This meta-analysis was conducted according to PRISMA guidelines [35].

\section{Results}

\section{Included studies}

We applied the PRISMA flow diagram to select the studies to be included in our meta-analysis [35]. We selected 1045 potentially relevant articles from eight databases. After deletion of duplicates, there were still 594 studies. By reviewing the titles and abstracts of these studies, we excluded 514 papers because they were obviously irrelevant. The full text of the remaining 80 articles was read, and another 48 studies were excluded (20 studies were

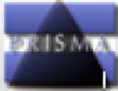

PRISMA 2009 Flow Diagram
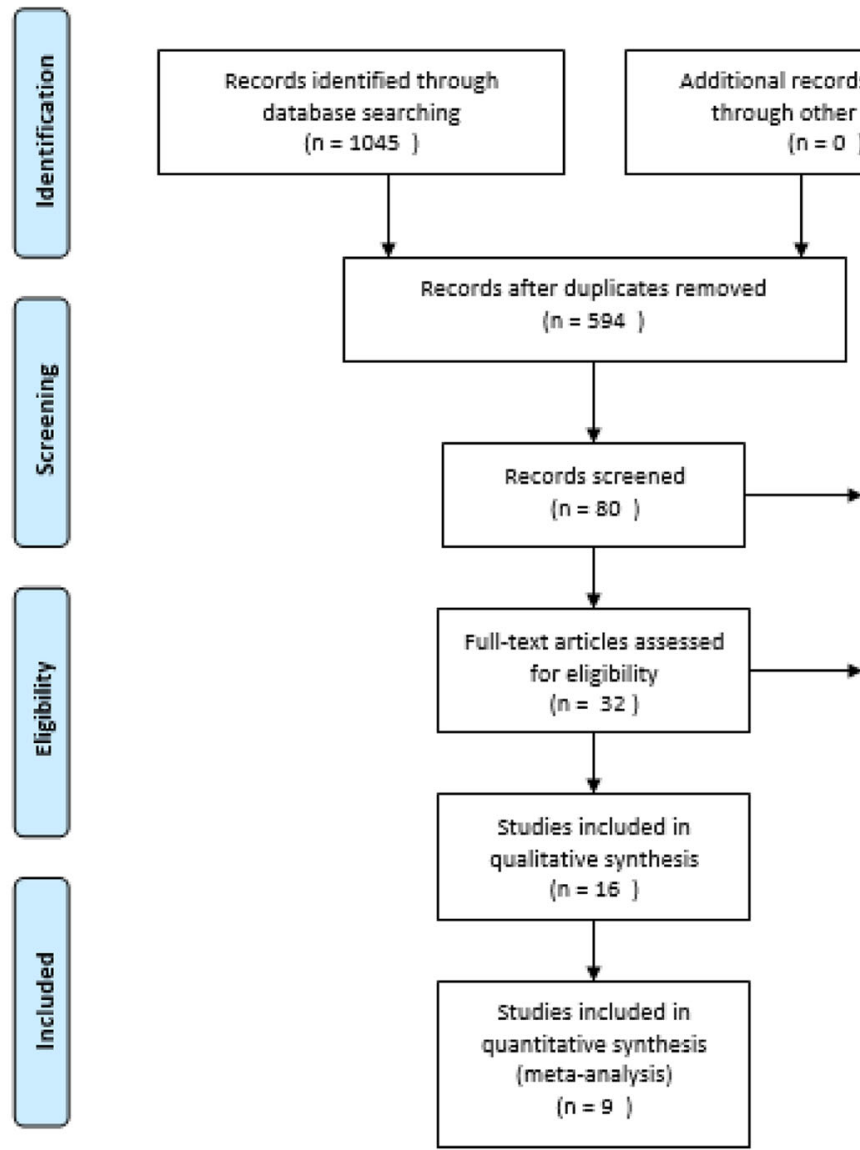

Fig. 1 Study selection flow chart 
not conducted in humans, and 28 studies were not clinical trials), yielding 32 studies. From these 32 studies, 16 studies were excluded (six were not case-control studies, three were not relevant to MCP-1, and seven were not relevant to OA). After removal of these studies, we included nine studies in this meta-analysis [36-44] (Fig. 1).

\section{Features of this study}

The nine studies included in this study described 376 patients with OA and 306 controls. The basic features of the studies are shown in Fig. 2. The testing method for MCP-1 levels in all nine studies was enzyme-linked immunosorbent assay (ELISA). Methodological quality assessment was performed using NOS, as shown in Fig. 2.

\section{Meta-analysis of $O A$}

Since there was significant heterogeneity among the nine studies $\left(p<0.00001, I^{2}=97 \%\right)$, a random-effects model was used. The results showed that MCP-1 levels were significantly higher in patients with OA than in controls $(\mathrm{SMD}=1.97,95 \% \mathrm{CI}=0.66-3.28, p=0.003$; Fig. 3). In particular, subgroup analysis showed that in both Asians and mixed populations, OA patients had higher MCP-1 serum levels than controls, whereas this result was not observed among Caucasians $(p>0.05)$. Moreover, MCP1 serum levels in patients with $\mathrm{OA}$ were significantly higher than those in the control group, but significant differences were not observed when MCP-1 levels were evaluated in the synovial fluid and cartilage tissue. Further subgroup analysis showed that MCP-1 levels were significantly higher in Chinese, Dutch, and Brazilian OA patients than in the respective controls; however, such differences were not observed in the American and Italian subgroups. Language of the study, sample size, and sample origin were not sources of heterogeneity because heterogeneity was still high after subgroup analysis (Figs. 4 and 5).

\section{Sensitivity analysis and publication bias}

The results of the sensitivity analysis indicated that none of the studies had an impact on the overall estimate of the association between MCP-1 levels and OA risk. Thus, our meta-analysis data were relatively stable and reliable (Fig. 6). Funnel plots of the nine included studies showed symmetry, and Egger's tests showed no publication bias ( $p=0.344$; Fig. 7).

\section{Discussion}

In this study, we assessed the relationship between MCP-1 expression and OA. Our meta-analysis showed that MCP-1 expression levels were significantly higher in patients with $\mathrm{OA}$ than in controls, indicating that $\mathrm{MCP}$ 1 was strongly correlated with the progression of OA. However, the specific mechanisms through which MCP1 influences OA remain unclear [45]. MCP-1 modulates chemokines expressed by monocytes and is one of the most important chemokines studied to date. Notably, binding of MCP-1 to receptors on the surface of OA chondrocytes can induce the production of matrix metalloproteinases (MMPs) and damage articular cartilage. Additionally, MCP-1 and RANTES promote cartilage catabolism, induce nitric oxide synthase, increase MMP3 expression, and inhibit proteoglycan synthesis $[8,46]$. MCP-1 chemotactic monocytes produce large numbers of pro-inflammatory cytokines in OA and promote the progression of OA [47]. Additionally, MCP-1 promotes the transformation of monocyte into macrophages and then to osteoclasts and can induce inflammation downstream [24, 48]. In previous studies, MCP-1 levels in serum and synovial fluid of patients with rheumatoid arthritis were found to be significantly increased

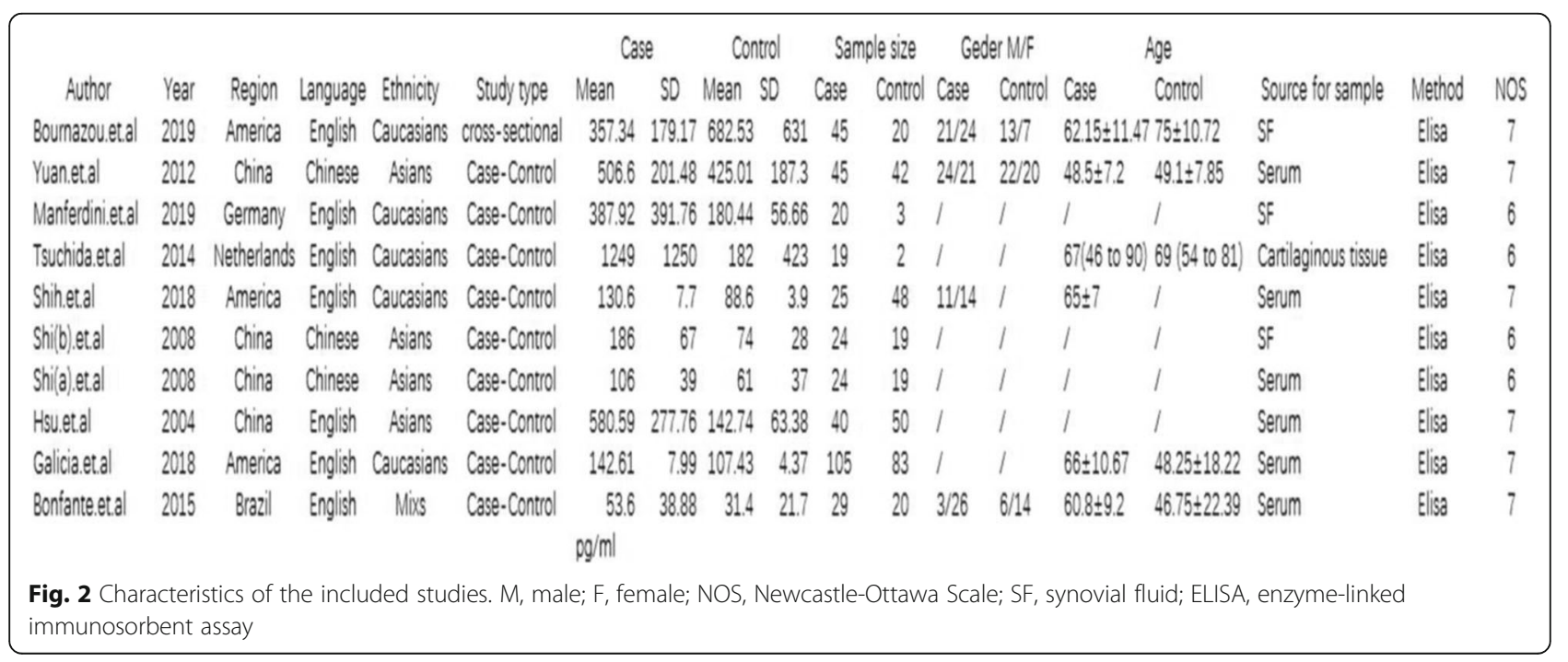




\begin{tabular}{|c|c|c|c|c|c|c|c|c|c|c|}
\hline \multirow[b]{2}{*}{ Studv or Subgroup } & \multicolumn{3}{|c|}{ Experimental } & \multicolumn{2}{|c|}{ Control } & \multicolumn{3}{|c|}{ Std. Mean Difference } & \multirow{2}{*}{\multicolumn{2}{|c|}{$\begin{array}{l}\text { Std. Mean Difference } \\
\text { V. Random, } 95 \% \mathrm{Cl}\end{array}$}} \\
\hline & Mean & SD & Iotal & Mean & SD & Total & Weight & IV. Random, $95 \% \mathrm{Cl}$ & & \\
\hline Bonfante 2015 & 53.6 & 38.88 & 29 & 31.4 & 21.7 & 20 & $10.3 \%$ & $0.66[0.08,1.25]$ & & 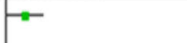 \\
\hline Bournazou 2019 & 357.34 & 179.17 & 45 & 682.5 & 631 & 20 & $10.3 \%$ & $-0.85[-1.40,-0.30]$ & $\rightarrow$ & \\
\hline Galicia 2018 & 142.61 & 7.99 & 105 & 107.4 & 4.37 & 83 & $10.2 \%$ & $5.28[4.67,5.89]$ & & - \\
\hline Hsu 2004 & 580.59 & 277.76 & 40 & 142.7 & 63.38 & 50 & $10.3 \%$ & $2.27[1.74,2.81]$ & & - \\
\hline Manferdini 2019 & 387.92 & 391.76 & 20 & 180.44 & 56.66 & 3 & $9.6 \%$ & $0.54[-0.69,1.76]$ & & \\
\hline Shi(a) 2008 & 106 & 39 & 24 & 61 & 37 & 19 & $10.2 \%$ & $1.16[0.50,1.81]$ & & $\rightarrow$ \\
\hline Shi(b) 2008 & 186 & 67 & 24 & 74 & 28 & 19 & $10.1 \%$ & $2.05[1.30,2.81]$ & & $\rightarrow$ \\
\hline Shih 2018 & 130.6 & 7.7 & 25 & 88.6 & 3.9 & 48 & $9.4 \%$ & $7.57[6.22,8.93]$ & & \\
\hline Tsuchida 2014 & 1,249 & 1,250 & 19 & 182 & 423 & 2 & $9.2 \%$ & $0.84[-0.64,2.32]$ & & \\
\hline Yuan 2012 & 506.6 & 201.48 & 45 & 425 & 187.3 & 42 & $10.4 \%$ & $0.42[-0.01,0.84]$ & & 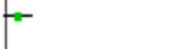 \\
\hline Total $(95 \% \mathrm{Cl})$ & & & 376 & & & 306 & $100.0 \%$ & $1.97[0.66,3.28]$ & & \\
\hline $\begin{array}{l}\text { Heterogeneity: Tau }{ }^{2} \\
\text { Test for overall effect }\end{array}$ & $\begin{array}{l}4.26 ; \mathrm{Chi}^{7} \\
\mathrm{Z}=2.94(\end{array}$ & $\begin{array}{l}i^{2}=342.0 \\
\langle P=0.003\end{array}$ & $3, d f=9$ & $9(P<0.0$ & $00001) ; r^{\prime}$ & $=97 \%$ & & & $\begin{array}{ccc} & -4 & 0 \\
-4 & -2 & 0 \\
\text { Favours (experimentall }\end{array}$ & $\begin{array}{lll}0 & 2 & 4 \\
\text { Favours [control] }\end{array}$ \\
\hline
\end{tabular}

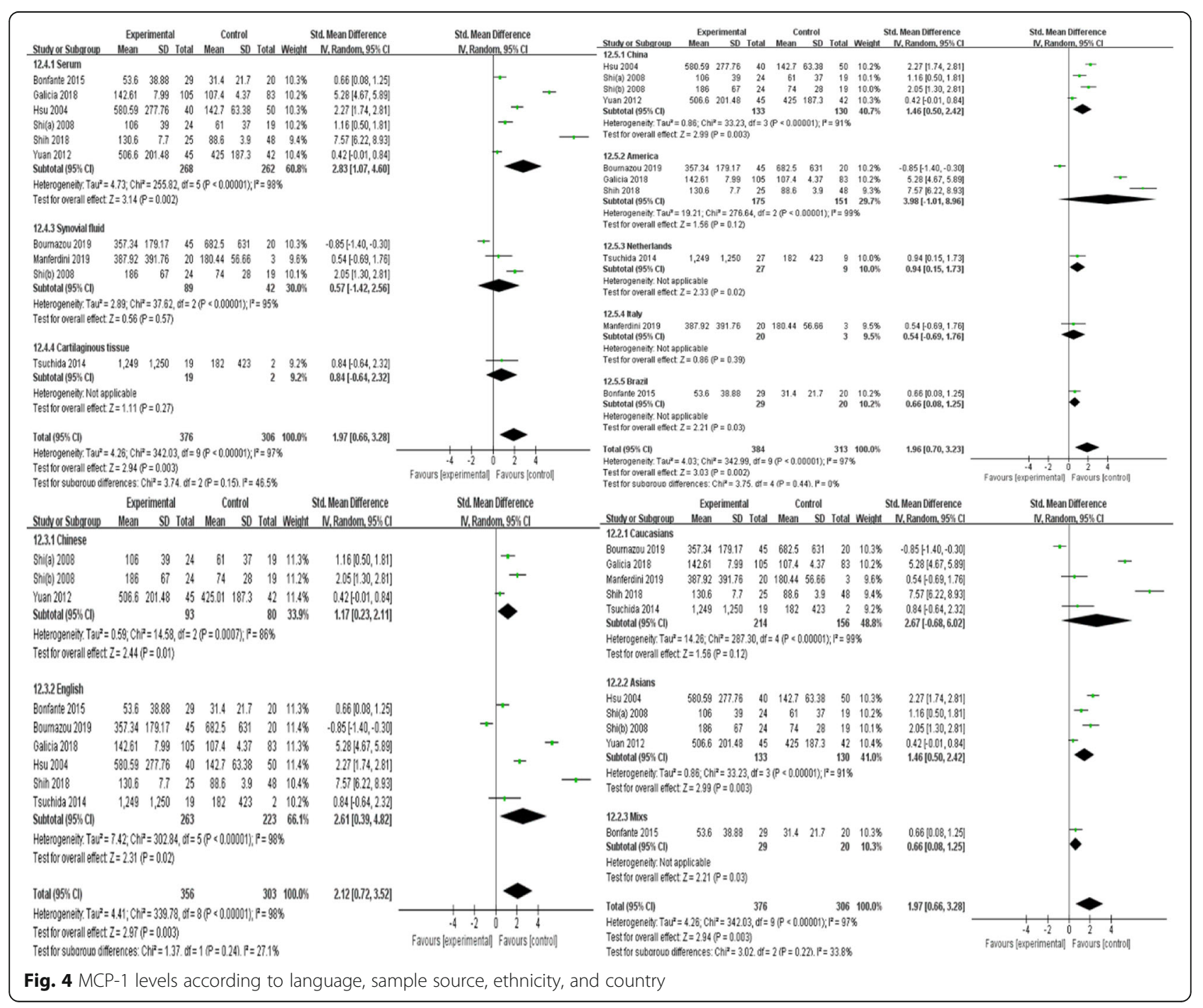




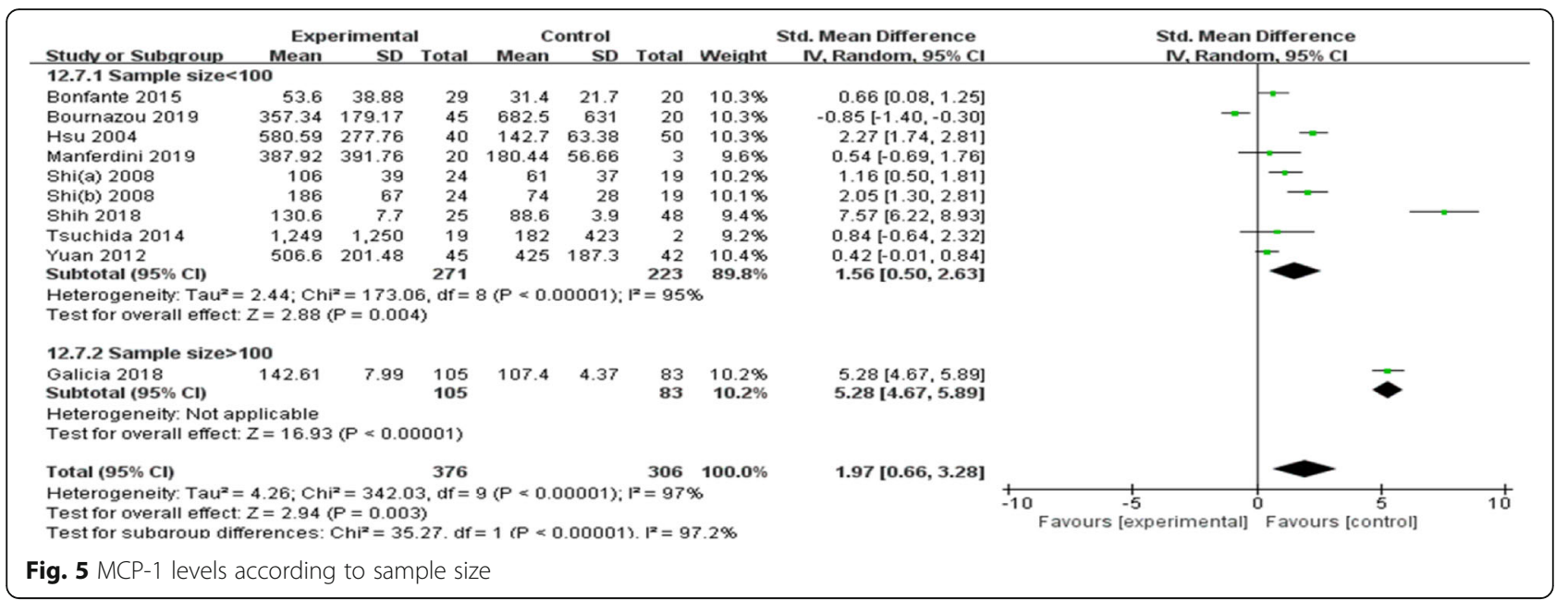

compared with that in healthy controls, and high levels of MCP-1 concentrated mononuclear cells from the blood to the articular cavity and then macrophages, activating downstream inflammatory responses and resulting in inflammatory injury [49-51]. Conversely, in a ccl2 knockout mouse model of OA, macrophages were found to accumulate less in OA tissues, and synovitis and cartilage damage were significantly reduced [14]. Interestingly, injection of MCP-1 into the knee joints of mice induced cartilage degradation, and in human studies, chondrocytes and synovial fibroblasts from patients with OA were found to express high levels of MCP-1. MCP-1 has also been shown to reflect the activity of macrophages and to be related to radiological findings and symptomatic inflammation in OA. Thus, MCP-1 may be a reliable indicator of disease severity [52-54]. Taken together, these findings demonstrated that MCP-1 plays important roles in the progression of $\mathrm{OA}$ and may contribute to the diagnosis of $\mathrm{OA}$, consistent with our results. Furthermore, MCP-1 levels are directly related to the grade of $\mathrm{OA}$, as more severe $\mathrm{OA}$ is associated with higher MCP-1 expression [55]. Therefore, MCP-1 may be applied as a potential biomarker to assess the severity of $\mathrm{OA}$ and as a target for clinical treatment.

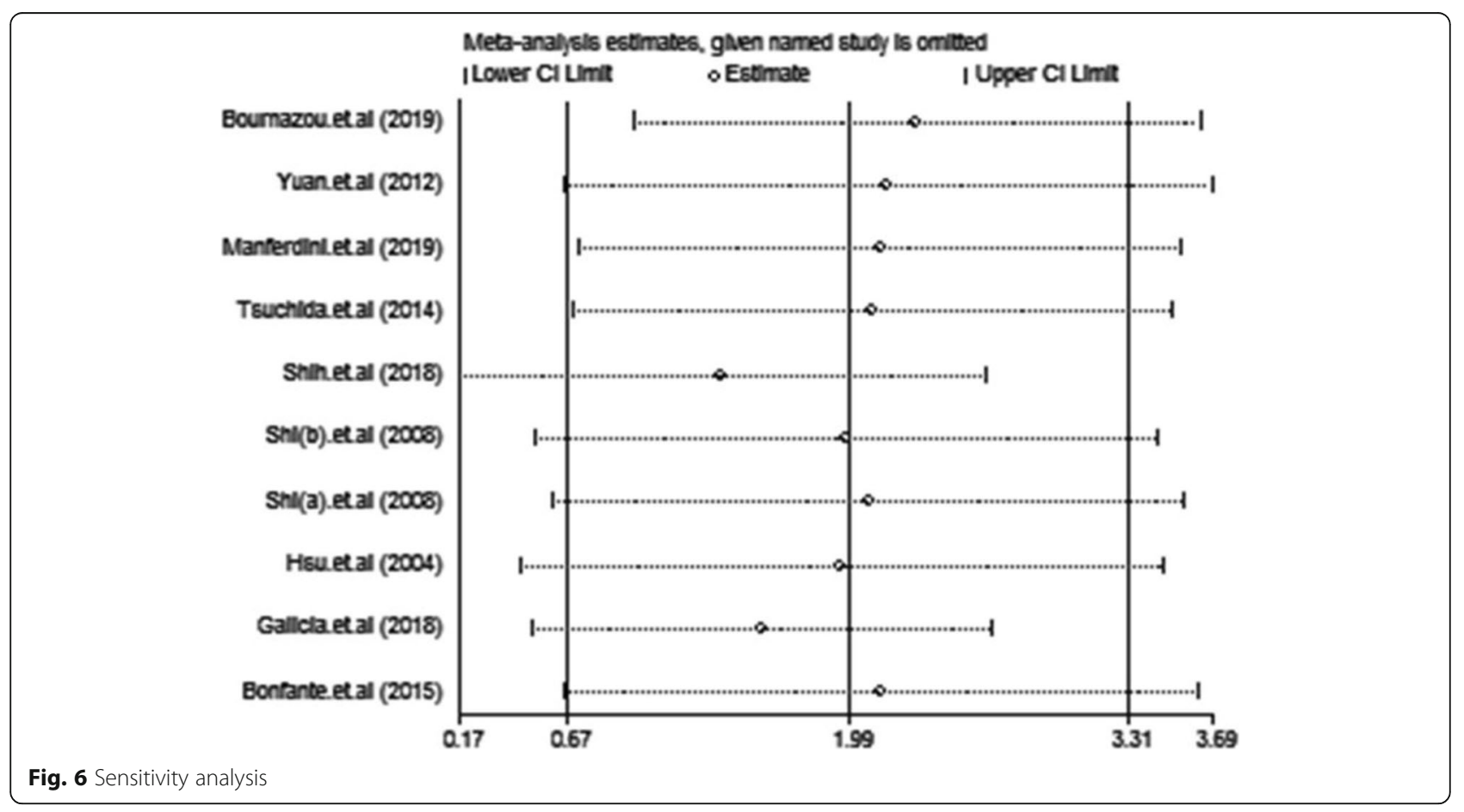




\section{Funnel plot with pseudo $95 \%$ confidence limits}

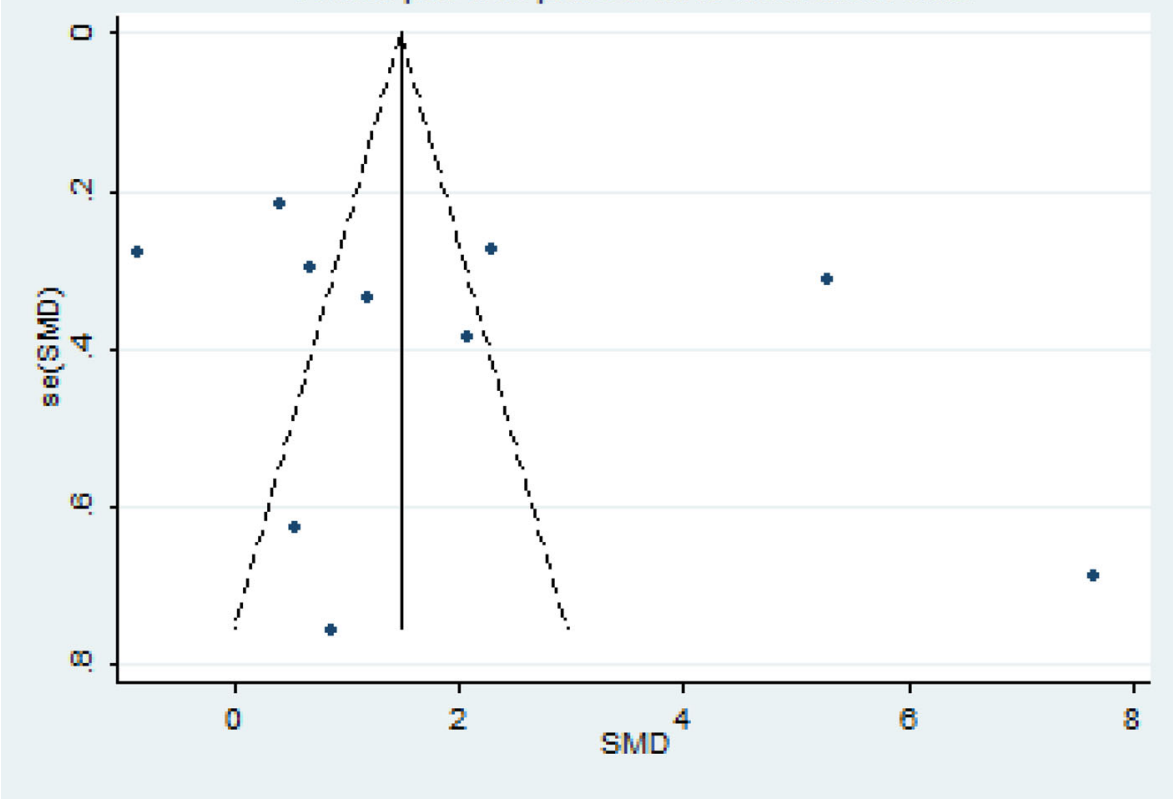

$\mathrm{t}=1.01, P=0.344$

Fig. 7 Funnel plots. $t=1.01, p=0.344$

To further explore the relationship between MCP-1 expression and OA progression and to examine possible sources of heterogeneity, we conducted a subgroup analysis based on language, ethnicity, country, and sample source. Ethnic subgroup analysis showed that MCP-1 serum levels were significantly higher in OA patients than in controls in Asian and mixed populations, but not in Caucasians. One possible explanation could be explained by the different physical qualities, lifestyles, eating habits, genetics, and environments of people belonging to these three ethnic groups. Moreover, country subgroup analysis showed significant differences in all subgroups except the USA and Italy. The results of the Italian subgroup could be explained by the fact that there were only three patients in the control group. Although the results for the two subgroups were not statistically significant, the data still showed higher MCP-1 expression in patients with $\mathrm{OA}$ than in the control group, suggesting a potential source of heterogeneity. In addition, a subgroup analysis based on language of the study and sample size showed that these were not sources of heterogeneity; nevertheless, future research should adjust these two factors to avoid heterogeneity in analyses. In contrast, sample source subgroup analysis showed that compared with healthy controls, MCP-1 expression was significantly increased in patients with OA when measured from serum samples, but not when evaluated using synovial fluid and cartilage tissue samples. This phenomenon may be related to the reduced quantity of samples for synovial fluid and cartilage or to the different characteristics of the control group and the fact that only one study was included in the cartilage tissue subgroup. Additionally, different sample sources may affect the diagnostic performance of the assay. Although studies have shown that the levels of MCP-1 in synovial fluid and joint soft tissues are significantly increased [54,56], such experiments are challenging due to ethical considerations; that is, it is not necessarily ethical to collect synovial fluid and cartilage tissues from healthy volunteers [57]. Thus, these factors may affect the reliability of the results. Therefore, this subgroup analysis confirmed the effectiveness of serum MCP-1 in the diagnosis of OA; however, further studies are needed to determine the correlation between MCP-1 levels in synovial fluid and cartilage tissue and $\mathrm{OA}$ severity.

The current meta-analysis has some limitations. First, the number of studies that were included in the analysis and their sample size were relatively small. Moreover, the lack of detailed data prevented to conduct subgroup analysis for other OA-related factors, such as sex, body mass index, and OA Kellgren and Lawrence grade, and this could affect the reliability of the results. Second, language may cause bias. Although there were no language restrictions when we searched the literature, the meta-analysis only included Chinese and English 
literature, this may lead to a degree of selective bias [58], but no bias was found when using Egger tests, indicating that the data obtained from the studies included in this meta-analysis are reliable and faithfully represent the reality. Third, the ELISA kits used in the studies are diverse and were purchased from different companies. Therefore, we could not determine the sensitivities of these kits. Finally, articles that provided only medians and ranges or upper and lower quartiles were excluded from the meta-analysis. Although a method for data conversion has been reported by Hozo et al. [59], we believe that if we forced the conversion of these data, the conversion results would not be accurate.

Despite the above limitations, this is the first metaanalysis to study the correlation between MCP-1 levels and OA. Thus, we used strict inclusion and exclusion criteria and applied appropriate statistical methods to combine the results of multiple studies in order to achieve strong objectivity, which suggested that our conclusions are reliable and meaningful.

Our results suggested that serum MCP-1 levels were closely related to $\mathrm{OA}$ and that $\mathrm{MCP}-1$ played important roles in the pathological progression of OA. At the same time, our results indicated that MCP-1 could be used as a potential biomarker for the diagnosis of $\mathrm{OA}$ and also as a therapeutic target for the treatment of OA. Given the limitations of the current study, additional rigorous and detailed experiments with large sample sizes are needed to verify our conclusions.

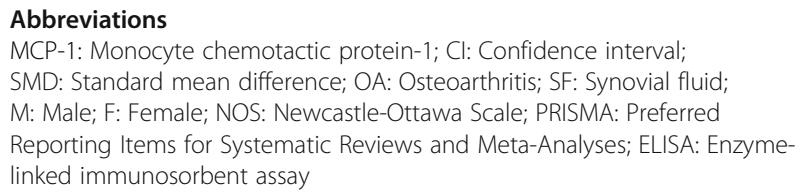

\section{Acknowledgements}

We would like to acknowledge the reviewers for their helpful comments on this paper.

\section{Authors' contributions}

Feifei Ni designed the study, carried out most of the data analysis, and wrote the manuscript; Yanchao Zhang, Xiaoxiao Peng, and Jianjun Li assisted with the preparation of the manuscript. The authors read and approved the final manuscript.

\section{Funding}

This work was supported by the National Natural Science Foundation of China (81971829).

\section{Ethics approval and consent to participate}

Because this article is a systematic review and meta-analysis, ethical approval was not required.

\section{Competing interests}

The authors declare that they have no competing interests.

\section{Author details}

'Department of Orthopaedics, Shengjing Hospital of China Medical University, Sanhao Street No. 36, Heping District, Shenyang 110004, Liaoning, People's Republic of China. ${ }^{2}$ Department of Orthopedics, Tianjin Baodi
Hospital/Baodi Clinical College of Tianjin Medical University, Tianjin 301800, People's Republic of China. ${ }^{3}$ Daxing Teaching Hospital of Capital Medical University, Beijing 102600, People's Republic of China.

Received: 28 July 2020 Accepted: 28 October 2020

Published online: 10 November 2020

\section{References}

1. Hawker GA. Osteoarthritis is a serious disease. Clin Exp Rheumatol. 2019; 37(Suppl 120(5)):3-6

2. Bortoluzzi A, Furini F, Scirè CA. Osteoarthritis and its management epidemiology, nutritional aspects and environmental factors. Autoimmun Rev. 2018;17(11):1097-104.

3. Plotnikoff R, et al. Osteoarthritis prevalence and modifiable factors: a population study. BMC Public Health. 2015;15:1195.

4. Mazor M, et al. Osteoarthritis biomarker responses and cartilage adaptation to exercise: a review of animal and human models. Scand J Med Sci Sports. 2019;29(8):1072-82.

5. Scanzello CR. Chemokines and inflammation in osteoarthritis: insights from patients and animal models. J Orthop Res. 2017;35(4):735-9.

6. Stein JV, Nombela-Arrieta C. Chemokine control of lymphocyte trafficking: a general overview. Immunology. 2005;116(1):1-12.

7. Wang $X$, et al. Metabolic triggered inflammation in osteoarthritis. Osteoarthr Cartil. 2015:23(1):22-30.

8. Endres $\mathrm{M}$, et al. Chemokine profile of synovial fluid from normal, osteoarthritis and rheumatoid arthritis patients: CCL25, CXCL10 and XCL1 recruit human subchondral mesenchymal progenitor cells. Osteoarthr Cartil. 2010;18(11):1458-66.

9. Borzi RM, et al. Human chondrocytes express functional chemokine receptors and release matrix-degrading enzymes in response to C-X-C and C-C chemokines. Arthritis Rheum. 2000;43(8):1734-41.

10. Lee MC, et al. CCL17 blockade as a therapy for osteoarthritis pain and disease. Arthritis Res Ther. 2018;20(1):62.

11. Nair A, et al. Synovial chemokine expression and relationship with knee symptoms in patients with meniscal tears. Osteoarthr Cartil. 2015;23(7): 1158-64.

12. Lisignoli $\mathrm{G}$, et al. Different chemokines are expressed in human arthritic bone biopsies: IFN-gamma and IL-6 differently modulate IL-8, MCP-1 and rantes production by arthritic osteoblasts. Cytokine. 2002;20(5):231-8.

13. Bilgic $H$, et al. Interleukin-6 and type I interferon-regulated genes and chemokines mark disease activity in dermatomyositis. Arthritis Rheum. 2009; 60(11):3436-46.

14. Raghu H, et al. CCL2/CCR2, but not CCL5/CCR5, mediates monocyte recruitment, inflammation and cartilage destruction in osteoarthritis. Ann Rheum Dis. 2017;76(5):914-22.

15. Dutta P, et al. MCP-1 is overexpressed in triple-negative breast cancers and drives cancer invasiveness and metastasis. Breast Cancer Res Treat. 2018; 170(3):477-86.

16. Salah SM, et al. MCP-1 promotes detrimental cardiac physiology, pulmonary edema, and death in the cpk model of polycystic kidney disease. Am J Physiol Renal Physiol. 2019;317(2):F343-f360

17. Liu S, et al. Increased serum MCP-1 levels in systemic vasculitis patients with renal involvement. J Interf Cytokine Res. 2018;38(9):406-12.

18. Talbert EE, et al. Circulating monocyte chemoattractant protein-1 (MCP-1) is associated with cachexia in treatment-naïve pancreatic cancer patients. J Cachexia Sarcopenia Muscle. 2018:9(2):358-68.

19. Lee WJ, et al. Plasma MCP-1 and cognitive decline in patients with Alzheimer's disease and mild cognitive impairment: a two-year follow-up study. Sci Rep. 2018;8(1):1280

20. Hoff $\mathrm{P}$, et al. Osteoarthritis synovial fluid activates pro-inflammatory cytokines in primary human chondrocytes. Int Orthop. 2013;37(1):145-51.

21. Lisignoli $\mathrm{G}$, et al. Chemokine expression by subchondral bone marrow stromal cells isolated from osteoarthritis (OA) and rheumatoid arthritis (RA) patients. Clin Exp Immunol. 1999;116(2):371-8.

22. Hampel $\mathrm{U}$, et al. Chemokine and cytokine levels in osteoarthritis and rheumatoid arthritis synovial fluid. J Immunol Methods. 2013;396(1-2):134-9.

23. Morrison NA, Day CJ, Nicholson GC. Dominant negative MCP-1 blocks human osteoclast differentiation. J Cell Biochem. 2014;115(2):303-12.

24. Villiger PM, Terkeltaub R, Lotz M. Monocyte chemoattractant protein-1 (MCP-1) expression in human articular cartilage. Induction by peptide 
regulatory factors and differential effects of dexamethasone and retinoic acid. J Clin Invest. 1992;90(2):488-96.

25. Gschwandtner M, Derler R, Midwood KS. More than just attractive: how CCL2 influences myeloid cell behavior beyond chemotaxis. Front Immunol. 2019;10:2759.

26. Kosek $\mathrm{E}$, et al. Differences in neuroimmune signalling between male and female patients suffering from knee osteoarthritis. J Neuroimmunol. 2018;321:49-60.

27. Miotla Zarebska J, et al. CCL2 and CCR2 regulate pain-related behaviour and early gene expression in post-traumatic murine osteoarthritis but contribute little to chondropathy. Osteoarthr Cartil. 2017;25(3):406-12.

28. Pantsulaia I, Kalichman L, Kobyliansky E. Association between radiographic hand osteoarthritis and RANKL, OPG and inflammatory markers. Osteoarthr Cartil. 2010;18(11):1448-53.

29. Chandran $V$, et al. Serum-based soluble markers differentiate psoriatic arthritis from osteoarthritis. Ann Rheum Dis. 2019;78(6):796-801.

30. Stang A. Critical evaluation of the Newcastle-Ottawa scale for the assessment of the quality of nonrandomized studies in meta-analyses. Eur J Epidemiol. 2010;25(9):603-5.

31. Zintzaras $E$, loannidis JP. Heterogeneity testing in meta-analysis of genome searches. Genet Epidemiol. 2005;28(2):123-37.

32. Higgins JP, Thompson SG. Quantifying heterogeneity in a meta-analysis. Stat Med. 2002;21(11):1539-58.

33. Song F, Gilbody S. Bias in meta-analysis detected by a simple, graphical test. Increase in studies of publication bias coincided with increasing use of meta-analysis. BMJ. 1998;316(7129):471.

34. Peters $\mathrm{J}$, et al. Comparison of two methods to detect publication bias in meta-analysis. JAMA. 2006;295(6):676-80.

35. Moher D, Liberati A, Tetzlaff J, Altman DG. Preferred reporting items for systematic reviews and meta-analyses: the PRISMA statement. PLoS Med. 2009;6(7):e1000097.

36. Bournazou E, et al. Vascular adhesion protein-1 (VAP-1) as predictor of radiographic severity in symptomatic knee osteoarthritis in the New York University cohort. Int J Mol Sci. 2019;20(11):2642.

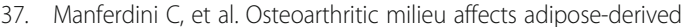
mesenchymal stromal cells. J Orthop Res. 2020;38(2):336-47.

38. Galicia K, et al. Inflammatory biomarker profiling in total joint arthroplasty and its relevance to circulating levels of lubricin, a novel proteoglycan. Clin Appl Thromb Hemost. 2018;24(6):950-9.

39. Shih $L$, et al. Postoperative changes in the systemic inflammatory milieu in older surgical patients. Clin Appl Thromb Hemost. 2018:24(4):583-8.

40. Bonfante HL, et al. CCL2, CXCL8, CXCL9 and CXCL10 serum levels increase with age but are not altered by treatment with hydroxychloroquine in patients with osteoarthritis of the knees. Int J Rheum Dis. 2017;20(12):1958-64.

41. Tsuchida Al, et al. Cytokine profiles in the joint depend on pathology, but are different between synovial fluid, cartilage tissue and cultured chondrocytes. Arthritis Res Ther. 2014;16(5):441.

42. Hsu YH, et al. Production of the chemokine eotaxin-1 in osteoarthritis and its role in cartilage degradation. J Cell Biochem. 2004;93(5):929-39.

43. Shangfeng $Y$. Levels of serum adiponection and its expression regulation by self-modified gubidingtong decoction in knee osteoarthritis patients. Chin J Trad Med Traumatol Orthop. 2012;20(01):41-3.

44. Qianfeng S, et al. Expression of RNATES, MCP-1 in serum and synovial fluid in patients with rheumatoid arthritis and its clinical significance. Modern J Integ Trad Chin Western Med. 2008;17(22):3410-2.

45. Chen WC, et al. Resistin enhances monocyte chemoattractant protein-1 production in human synovial fibroblasts and facilitates monocyte migration. Cell Physiol Biochem. 2019;52(3):408-20.

46. Honorati $\mathrm{MC}$, et al. Contribution of interleukin 17 to human cartilage degradation and synovial inflammation in osteoarthritis. Osteoarthr Cartil. 2002;10(10):799-807.

47. Loukov D, et al. Monocyte activation is elevated in women with kneeosteoarthritis and associated with inflammation, BMI and pain. Osteoarthr Cartil. 2018;26(2):255-63.

48. Villiger PM, Terkeltaub R, Lotz M. Production of monocyte chemoattractant protein-1 by inflamed synovial tissue and cultured synoviocytes. J Immunol. 1992;149(2):722-7.

49. Szekanecz Z, Kim J, Koch AE. Chemokines and chemokine receptors in rheumatoid arthritis. Semin Immunol. 2003;15(1):15-21.

50. Elemam NM, Hannawi S, Maghazachi AA. Role of chemokines and chemokine receptors in rheumatoid arthritis. Immunotargets Ther. 2020; 9:43-56.
51. Rudolph EH, Woods JM. Chemokine expression and regulation of angiogenesis in rheumatoid arthritis. Curr Pharm Des. 2005;11(5):613-31.

52. Xu YK, et al. The role of MCP-1-CCR2 ligand-receptor axis in chondrocyte degradation and disease progress in knee osteoarthritis. Biol Res. 2015;48:64

53. Haraden CA, et al. Synovial fluid biomarkers associated with osteoarthritis severity reflect macrophage and neutrophil related inflammation. Arthritis Res Ther. 2019;21(1):146.

54. Li L, Jiang BE. Serum and synovial fluid chemokine ligand $2 /$ monocyte chemoattractant protein 1 concentrations correlates with symptomatic severity in patients with knee osteoarthritis. Ann Clin Biochem. 2015;52(Pt 2):276-82.

55. Longobardi $L$, et al. Associations between the chemokine biomarker CCL2 and knee osteoarthritis outcomes: the Johnston County Osteoarthritis Project. Osteoarthr Cartil. 2018;26(9):1257-61.

56. Favero $\mathrm{M}$, et al. Inflammatory molecules produced by meniscus and synovium in early and end-stage osteoarthritis: a coculture study. J Cell Physiol. 2019;234(7):11176-87.

57. Hao HQ, et al. Cartilage oligomeric matrix protein, C-terminal cross-linking telopeptide of type II collagen, and matrix metalloproteinase-3 as biomarkers for knee and hip osteoarthritis (OA) diagnosis: a systematic review and meta-analysis. Osteoarthr Cartil. 2019;27(5):726-36.

58. Guyatt GH, et al. GRADE guidelines: 5. Rating the quality of evidence-publication bias. J Clin Epidemiol. 2011;64(12):1277-82.

59. Hozo SP, Djulbegovic B, Hozo I. Estimating the mean and variance from the median, range, and the size of a sample. BMC Med Res Methodol. 2005;5:13.

\section{Publisher's Note}

Springer Nature remains neutral with regard to jurisdictional claims in published maps and institutional affiliations.
Ready to submit your research? Choose BMC and benefit from:

- fast, convenient online submission

- thorough peer review by experienced researchers in your field

- rapid publication on acceptance

- support for research data, including large and complex data types

- gold Open Access which fosters wider collaboration and increased citations

- maximum visibility for your research: over $100 \mathrm{M}$ website views per year

At BMC, research is always in progress.

Learn more biomedcentral.com/submissions 